\title{
A Clinical and Radiological Approach to the Management of Benign Mesenchymal Sinonasal Tumors
}

\author{
Lukas Anschuetz $^{a} \quad$ Melanie Buchwalder ${ }^{a}$ Matthias Dettmer ${ }^{b}$ \\ Marco D. Caversaccio ${ }^{a}$ Franca Wagner ${ }^{c}$ \\ a University Department of Otorhinolaryngology, Head and Neck Surgery, Inselspital Bern, \\ University Hospital and University of Bern, ${ }^{b}$ Department of Clinical Pathology, Institute of \\ Pathology, University of Bern, and ' University Department of Diagnostic and Interventional \\ Neuroradiology, Inselspital Bern, University Hospital and University of Bern, Bern, Switzerland
}

\section{Keywords}

Sinonasal mass · Benign mesenchymal sinonasal neoplasms · Osteoma · Fibrous dysplasia .

Computed tomography · Magnetic resonance imaging

\begin{abstract}
Purpose: Benign mesenchymal sinonasal neoplasms (BMSN) are rare and histologically heterogeneous. Differential diagnosis, appropriate management, and outcome are still a matter of debate. The aim of this study is to provide evidence for further refinement of assessment and treatment in the future. Procedures: We retrospectively reviewed data on 93 patients with neuroradiologically verified BMSN treated at our university reference center during the past 22 years. Results: The most frequent BMSN recorded in our cohort was osteoma of the frontal sinus. Only one-third of the patients affected were symptomatic at initial presentation. The 2 other common fibro-osseous tumor entities, fibrous dysplasia and ossifying fibroma, were confirmed in 12 and 6 patients, respectively. Patients with soft tissue tumor entities such as hemangioma, glomangiopericytoma, angiofibroma, and hamartoma were all symptomatic and underwent surgical resection. Conclusion: Understanding and recognizing the spectrum of appearances of benign mesenchymal sinonasal tumors will improve patient assessment and clinical management. The pathognomonic neuroradiological signs of a particular tumor entity should be actively sought as the neuroradiological features may be the diagnostic clues. Computed tomography and magnetic resonance imaging play complementary roles in identifying the morphological details and locoregional staging of benign mesenchymal sinonasal tumors.




\section{Introduction}

The management of benign mesenchymal sinonasal neoplasms (BMSN) is a clinically challenging aspect of otorhinolaryngology. The initial clinical presentation of patients with BMSN is unspecific; the most often documented symptoms being headache, nasal obstruction, and discharge [1]. Concomitant infection caused by the obstruction of the nasal drainage pathways and abundant inflammatory tissue may hinder a straightforward diagnosis and an underlying neoplasm can remain occult for years [2]. The differential diagnosis is based on endoscopic findings and neuroradiological appearance. When rhinological symptoms are persistent, computed tomography (CT) and, based on the CT findings, optional magnetic resonance imaging (MRI), are indicated. The diagnostic workup may be completed by a biopsy [3].

In 1948, Willis classified benign sinonasal neoplasms according to their histological derivation into epithelial, mesenchymal, and neural tumors [4]. The World Health Organization Classification of Tumors introduced further subdivisions into epithelial, soft tissue, bone and cartilage, hematolymphoid, neuroectodermal and germ cell tumors [5]. BMSNs therefore exhibit considerable histological heterogeneity. The most frequently encountered BMSNs are fibro-osseous lesions such as osteoma, fibrous dysplasia (FD), and ossifying fibroma (OF). Histologically and neuroradiologically documented BMSNs of soft tissue or hematolymphoid tumors like angiofibroma, hemangioma, glomangiopericytoma (GPC), hamartoma, and paraganglioma are rarely documented [5].

With CT and MRI diagnosis verification, tissue characterization, and description of expansion are possible and can contribute to optimize the endoscopic surgical treatment of sinonasal tumors [6]. Besides the benign histological aspect of BMSNs, the lesions may have intracranial and orbital complications [7-11]. Evidence on optimal treatment is still scarce, and further knowledge about the natural evolution and response to different treatment modalities of BMSN is required.

CT and MRI offer certain advantages, and each has disadvantages when compared with other techniques. Notably, plain films are no longer considered to be a part of the primary imaging protocol [12]. Classically, benign neoplasms expand and remodel the bone, whereas aggressive malignancies destroy and invade adjacent tissues with ill-defined margins. CT has superior bony definition, whereas MRI is better at distinguishing between tumor and retained secretions, thus offering improved assessment of tumor extent and assisting in choosing adequate access to surgery. MRI gives superior soft tissue delineation in the adjacent infratemporal fossa, masticator space, and in evaluation of perineural, intraorbital and intracranial tumor spread [13]. Imaging is vital in distinguishing tumors from infection, retained secretions, and granulation scar tissue. Even if imaging is performed in the early stages, a neuroradiologist inexperienced in sinonasal anatomy and tumor features may interpret early signs of a tumor as rhinosinusitis or a lesion that does not require follow-up. The intention of this article is to describe and review the clinical and neuroradiological imaging findings in patients with BMSN documented in our tertiary care center in the past 22 years.

\section{Patients and Methods}

Ethical Approval

This retrospective study was approved by the Ethics Committee of the Canton of Bern and was performed in accordance with the Declaration of Helsinki [14].

Patient Selection

We conducted a retrospective review of 129 patients whose neuroradiological report led to primary suspicion of BMSN. Overall, 177 images of these patients (CT and/or MRI) were acquired between 1994 and 
Anschuetz et al.: A Clinical and Radiological Approach to the Management of Benign Mesenchymal Sinonasal Tumors

Table 1. Summary of case distribution, imaging modalities, surgical treatment, and tumor extension

\begin{tabular}{|c|c|c|c|c|c|c|c|}
\hline & Osteoma & $\begin{array}{l}\text { Fibrous } \\
\text { dysplasia }\end{array}$ & $\begin{array}{l}\text { Ossifying } \\
\text { fibroma }\end{array}$ & $\begin{array}{l}\text { Hemangioma } \\
\text { GPC }\end{array}$ & Angiofibroma & Hamartoma & "Other ${ }^{a "}$ \\
\hline Patients & 48 & 12 & 6 & 9 & 8 & 3 & 7 \\
\hline Sex & $\begin{array}{l}\text { F: } 16(33 \%) \\
\text { M: } 32(67 \%)\end{array}$ & $\begin{array}{l}\text { F: } 5(42 \%) \\
\text { M: } 7(58 \%)\end{array}$ & $\begin{array}{l}\text { F: } 3(50 \%) \\
\text { M: } 3(50 \%)\end{array}$ & $\begin{array}{l}\text { F: } 5(56 \%) \\
\text { M: } 4(44 \%)\end{array}$ & $\begin{array}{l}\text { F: } 0(0 \%) \\
\text { M: } 8(100 \%)\end{array}$ & $\begin{array}{l}\text { F: } 3(100 \%) \\
\text { M: } 0(0 \%)\end{array}$ & $\begin{array}{l}\text { F: } 4(57 \%) \\
\text { M: } 3(43 \%)\end{array}$ \\
\hline Mean age, years & 45 & 38 & 38 & 50 & 23 & 55 & 38 \\
\hline CT & 48 & 12 & 6 & 9 & 8 & 3 & 7 \\
\hline MRI & 5 & 6 & 4 & 5 & 8 & 1 & 4 \\
\hline Predominant localization & $\begin{array}{l}\text { Frontal } \\
(n=34,71 \%)\end{array}$ & $\begin{array}{l}\text { Sphenoid } \\
(n=6,50 \%)\end{array}$ & $\begin{array}{l}\text { Frontal } \\
(n=3,50 \%)\end{array}$ & $\begin{array}{l}\text { Nasal cavity } \\
(n=7,78 \%)\end{array}$ & $\begin{array}{l}\text { Multiple } \\
(n=5,63 \%)^{\mathrm{b}}\end{array}$ & $\begin{array}{l}\text { Nasal cavity } \\
(n=2,66 \%)\end{array}$ & $\begin{array}{l}\text { Sphenoid } \\
(n=2,29 \%)\end{array}$ \\
\hline Skull base involvement & - & 5 & 2 & - & 1 & 1 & 1 \\
\hline Orbital involvement & 2 & 1 & 1 & - & - & - & 1 \\
\hline Symptomatic cases & $17(35 \%)$ & $10(84 \%)$ & $4(66 \%)$ & $9(100 \%)$ & $8(100 \%)$ & $3(100 \%)$ & $7(100 \%)$ \\
\hline \multicolumn{8}{|l|}{ Surgical treatment ( $n=48,52 \%)$} \\
\hline Endoscopic & 1 & - & 1 & $4^{\mathrm{c}}$ & $5^{\mathrm{d}, \mathrm{e}}$ & 3 & 3 \\
\hline Open & 7 & 1 & 3 & $2^{\mathrm{f}}$ & $3^{\mathrm{d}}$ & - & 4 \\
\hline Combined & 4 & 1 & & - & & - & \\
\hline Biopsy & 1 & 2 & - & - & & - & \\
\hline $\begin{array}{l}\text { Biopsy + functional endoscopic } \\
\text { sinus surgery }\end{array}$ & 1 & 2 & - & - & & - & \\
\hline
\end{tabular}

${ }^{a}$ Two giant cell tumors, 1 osteochondroma, 1 myxoma, 1 fibromyxoma, 2 cholesterol granulomas. ${ }^{\mathrm{b}} \mathrm{CO}_{2}$ laser. ${ }^{\mathrm{c}}$ One preoperative embolization, 2 additional exclusive embolizations. ${ }^{\mathrm{d}}$ Prior embolization in all cases. ${ }^{\mathrm{e}} 1$ adjuvant radiotherapy. ${ }^{\mathrm{f}}$ Nasopharynx involvement $n=7$.

2015. The documented clinical data, the histological reports and all imaging series acquired from the initially selected 129 patients were reviewed. Altogether, 36 patients had to be excluded: (a) due to the postoperative histologically proven epithelial origin of the sinonasal tumor mass, e.g. inverted papilloma or inflammatory polyp, (b) incomplete treatment, or (c) incomplete follow-up datasets. A total of 93 patients finally fulfilled the inclusion criteria and were analyzed retrospectively. For the 47 symptom-free patients included in the study, a "wait and scan" policy was adopted in the case of an unequivocal neuroradiological diagnosis.

\section{Imaging Analysis}

Qualitative evaluation of CT and MRI scans was performed by an experienced neuroradiologist (F.W.). All CT and MRI scans were validated on a certified reporting station (DIN V 6868-57 and quality assurance guideline).

\section{CT and MRI}

All patients $(100 \%)$ had a CT examination of the viscerocranium. All imaging studies were performed using our CT scanner (Somatom Definition Edge) with high-resolution bone kernel (slice thickness [ST] $1 \mathrm{~mm}$ and field of view $200 \mathrm{~mm}$ ) and soft tissue algorithm with optional contrast application depending on the clinical question. A 3D reformation was done in all cases. Thirty-three (35.5\%) of the 93 patients underwent an additional MRI of the head and neck for exact determination of the tumor extent (Table 1). The MRI data sets were acquired using a 1.5-T or a 3-T Siemens scanner (Magnetom Avanto or Magnetom Verio, respectively; Siemens Medical Solutions, Erlangen, Germany) with 12-channel head coils. Our standard MRI protocol for the evaluation of pathologies of the viscerocranium for both scanner types included the following native sequences: axial T1-weighted (T1w; ST $5 \mathrm{~mm}$ ) and T2-weighted (T2w; ST $5 \mathrm{~mm}$ ) sequences for the whole brain, coronal T2w (ST $3 \mathrm{~mm}$ ) and coronal turbo inversion recovery magnitude (ST $4 \mathrm{~mm}$ ) covering the viscerocranium, and an axial T1w (ST $3 \mathrm{~mm}$ ) over the viscerocranium. The following were acquired after contrast application: coronal T1w with fat suppression (ST $3 \mathrm{~mm}$ ) covering the viscerocranium, axial T1w with fat suppression (ST $3 \mathrm{~mm}$ ) above the viscerocranium, and 3D T1w multiplanar (MPR) sequences (ST 1 $\mathrm{mm}$ ) over the whole brain. 
Table 2. Clinical presentation of patients according to tumor localization

\begin{tabular}{|c|c|c|c|c|c|c|c|c|}
\hline & $\begin{array}{l}\text { Nasal } \\
\text { obstruction }^{\text {a }}\end{array}$ & CRS & Epistaxis & $\begin{array}{l}\text { External } \\
\text { swelling }\end{array}$ & Pain & $\begin{array}{l}\text { Proptosis/ } \\
\text { diplopia }\end{array}$ & Incidental & Total \\
\hline \multicolumn{9}{|l|}{ Nasal fossa and } \\
\hline epipharynx & 9 & - & 3 & 3 & - & - & 1 & 16 \\
\hline Maxillary sinus & 2 & 2 & - & 3 & 1 & - & - & 8 \\
\hline Frontal sinus & - & 10 & - & 1 & 5 & 1 & 21 & 38 \\
\hline Ethmoidal sinus & - & 2 & - & - & - & 1 & 8 & 11 \\
\hline Sphenoid sinus & 2 & 2 & - & - & 4 & 1 & 4 & 13 \\
\hline Multiple & 4 & 1 & & 1 & & & 1 & 7 \\
\hline Total & 17 & 17 & 3 & 8 & 10 & 3 & 35 & 93 \\
\hline
\end{tabular}

\section{Results}

Ninety-three patients (36 females and 57 males) aged 10-87 years (average 42 years) were retrospectively analyzed. The case distribution and imaging modalities are summarized in Table 1. The localization of the BMSN according to the leading clinical symptom is documented in Table 2. The 93 patients were followed up for a mean of 55 months, ranging from 0 months (incidental tumor without follow-up) to 33 years.

The indications for conservative or surgical treatment were discussed individually for every patient and depended on the clinical presentation and the response to conservative treatments. The decision to undertake surgical tumor resection depended on the tumor size, localization of the tumor, and the preoperative imaging diagnosis. Six of the 93 patients $(6.5 \%)$ underwent a primary biopsy for diagnostic purposes due to inconclusive imaging findings.

The most frequent BMSN encountered was osteoma $(n=48,52 \%)$, mostly located in the frontal sinus (34 patients, 71\%; Fig. 1). Only one-third of those patients were symptomatic at initial presentation. In the two-thirds of the patients who were asymptomatic, the osteoma was described as an incidental finding during radiological studies. A surgical resection was performed in 14 of the 48 patients (29.2\%).

The 2 other common fibro-osseous tumor entities, FD (Fig. 2) and OF (Fig. 3), were confirmed in $12(12.9 \%)$ and $6(6.5 \%)$ patients, respectively. Of the subgroup of FD patients, $84 \%$ (10 patients) were symptomatic, compared to $66 \%$ (4 patients) in the OF group. Six patients (50\%) underwent surgical therapy for FD, whereas for OF, 4 patients $(66.6 \%)$ underwent surgery.

The rare soft tissue tumor entities such as hemangioma (6 patients, 6.5\%; Fig. 4), GPC (3 patients, 3.2\%; Fig. 5), angiofibroma (8 patients, 8.6\%; Fig. 6), and hamartoma (3 patients, $3.2 \%$; Fig. 7) were symptomatic in all cases and treated with surgical resection. In our study, we also observed some diagnostic sinonasal BMSN rarities: 2 giant cell tumors (2.2\%; Fig. 8); 1 osteochondroma (1.1\%), 1 myxoma (1.1\%), 1 fibromyxoma (1.1\%; Fig. 9) and 2 cholesterol granulomas (2.2\%; Fig. 10). These patients were all symptomatic and therefore surgically treated.

Overall, 48 (52\%) of the 93 patients studied underwent surgical resection; in 42 (87.5\%) of the patients who were operated on, a complete tumor resection was achieved. Three patients (3.2\%) underwent functional endoscopic sinus surgery leaving the main tumor mass in situ. Two patients $(2.2 \%)$ presented with an extended hemangioma exclusively treated by 
Fig. 1. Two examples of CT of small osteomas. a-c In the first patient, the CT scan of the paranasal sinus in axial, coronal, and sagittal reformations shows a small ossified mass with smooth borders in the left posterior ethmoidal sinus adjacent to the bone, with a broad-based stalk; classic image of an incidental osteoma. d, e In the second patient, the CT of the paranasal sinus also shows an incidental finding of a classic ovoid osteoma with a small stalk in the left frontal sinus.
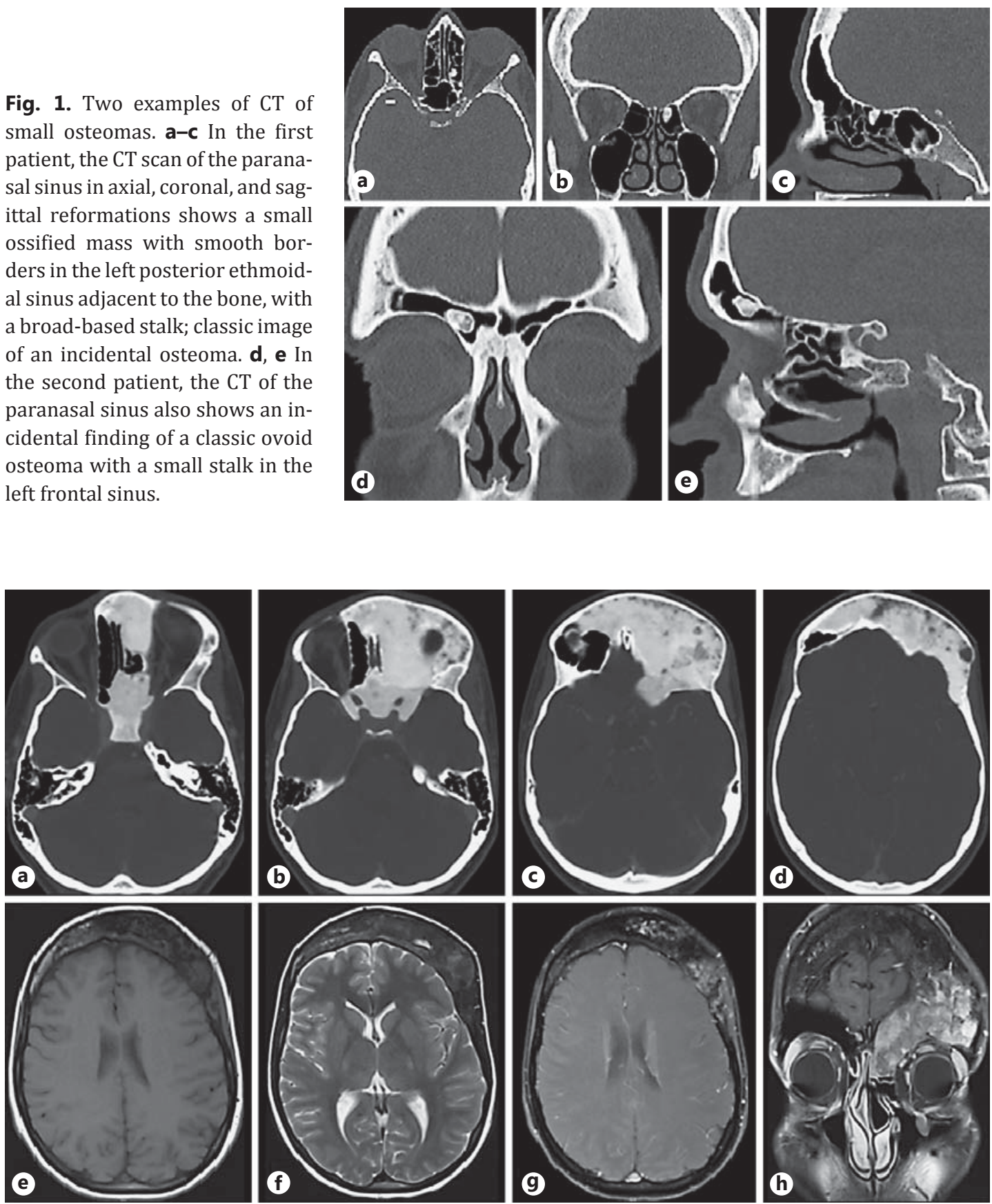

Fig. 2. The axial CT scan (bone window, a-d) and the MRI (e-h) of the brain and paranasal sinus reveal massive craniofacial fibrous dysplasia (FD) with a classic ground glass pattern on the CT scan. On MRI, the FD is classically inhomogeneous and hypointense on T1w (e), inhomogeneous and hypo- to hyperintense on T2w (f) with avid heterogeneous enhancement after contrast application $(\mathbf{g}, \mathbf{h})$, indicating an active state.

tumor embolization. Among the 48 patients who underwent a surgical procedure, peri-interventional complications were reported in $9(18.75 \%)$. The surgical details are summarized in Table 3.

Follow-up evaluation showed that osteomas were prone to relapse or growth of residual tumor after primary resection. In total, we observed 6 (42.9\%) tumor relapses and 3 (21.4\%) 
Fig. 3. CT scan of the paranasal sinus with primary contrast application; bone (a) and soft tissue (b) window with reformatted coronal (c) and sagittal plane (d). The CT demonstrates an expansive mass-like lesion in the right cavity of the maxillary sinus with remodeling of the sinus wall and adjacent reactive hyperostosis, suggestive of an ossifying fibrosis in its early stage.

Fig. 4. Native CT scan of the paranasal sinus; bone (a) and soft tissue (b) window with reformation (coronal, c; sagittal, d). Small expansive lesion with a honeycomb trabeculated pattern located at the anterior nasal bone on the left side with remodeling but no erosion of the adjacent intact cortical lamella. On imaging, the diagnosis is compatible with a hemangioma.
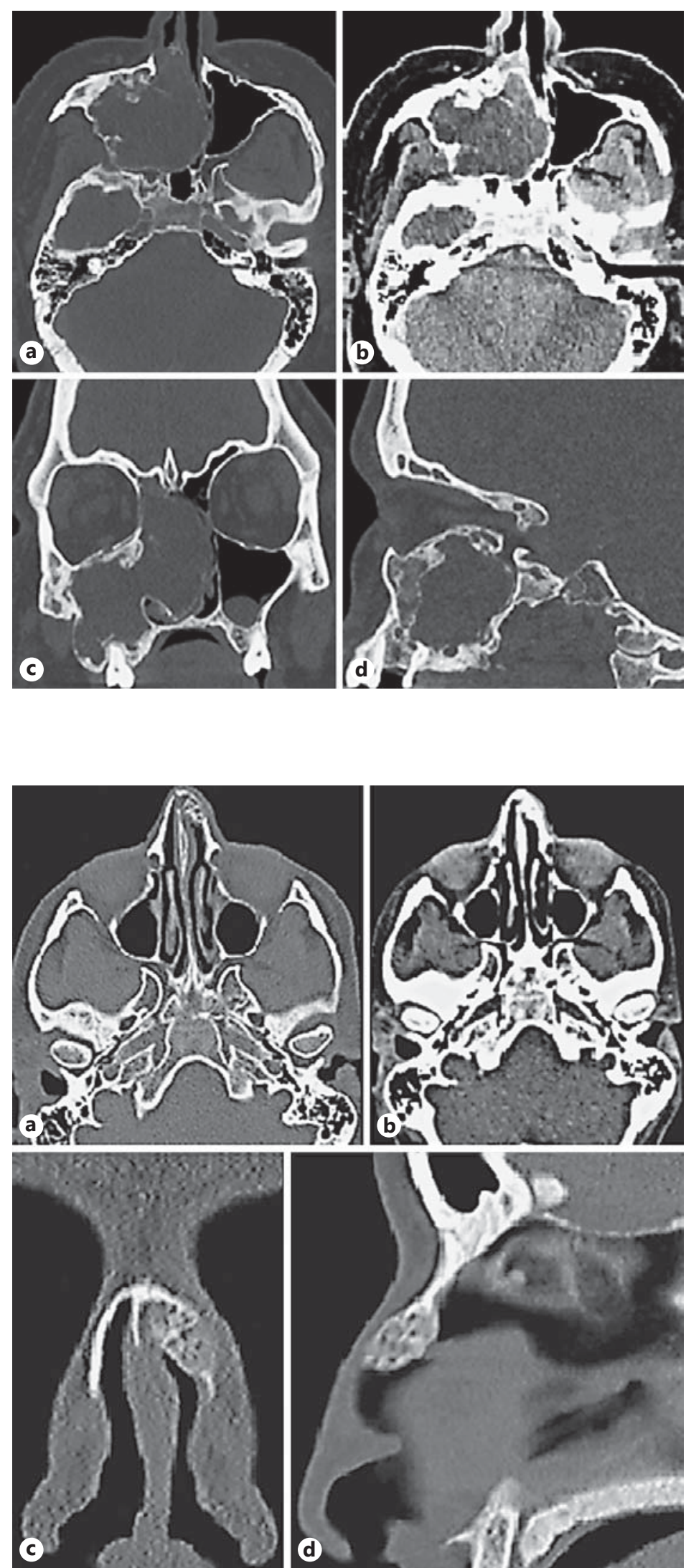
Fig. 5. CT scan of the paranasal sinus; native (bone window, a; soft tissue window, b) and after contrast application (axial, c; sagittal, d). Centered in the right nasal cavity is a polypoid smooth-bordered soft tissue mass with avid and, because of its size, inhomogeneous enhancement. The nasal septum seems to be remodeled but not destroyed. This was later histologically proven to be a glomangiopericytoma.

Fig. 6. The MRI of a young male patient with recurrent epistaxis and nasal obstruction exhibits a low-intensity expansive soft tissue mass on T1w (a), inhomogeneous on $\mathrm{T} 2 \mathrm{w}$ (b) images with avid inhomogeneous enhancement $(\mathbf{c}, \mathbf{d})$ centered in the sphenopalatine foramen on the right with tumor extension into the pterygopalatine fossa, buccal space, nasal cavity and nasopharynx and central skull base with already visible destruction of clivus and encasement of the internal right carotid artery. In correlation with the clinical state, imaging shows that the lesion is likely to be a juvenile angiofibroma.
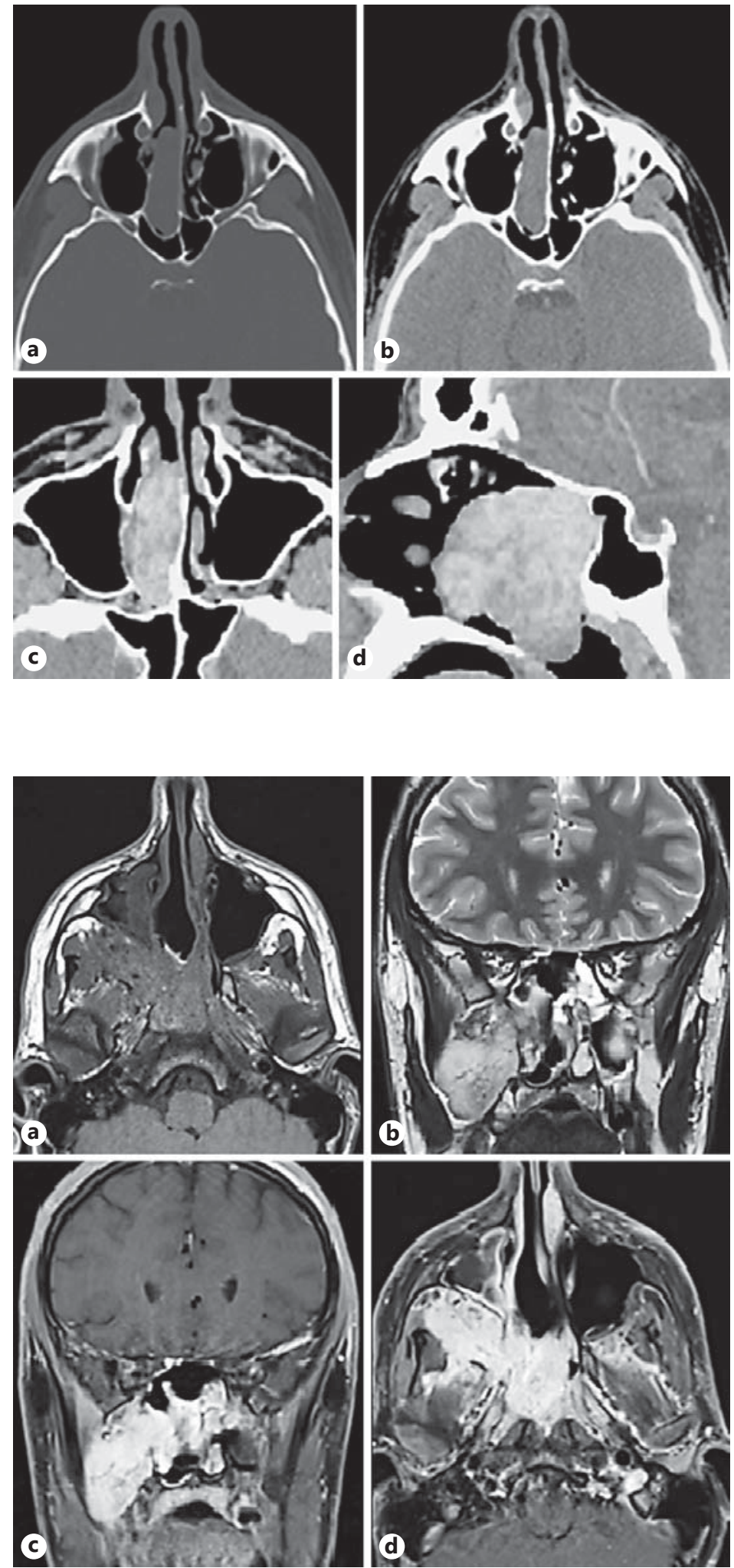

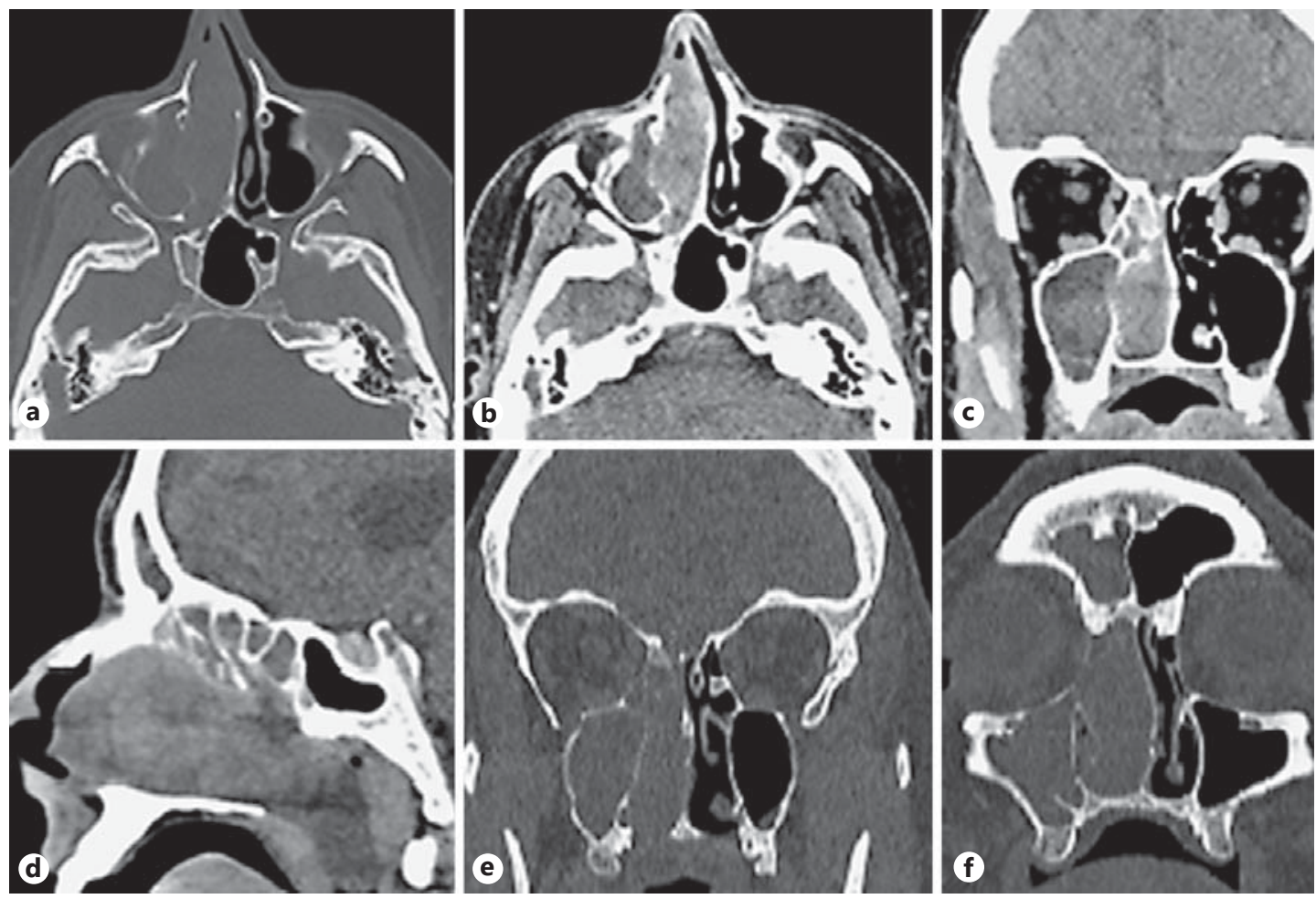

Fig. 7. CT scan of the paranasal sinus with primary contrast application; axial bone window (a), soft tissue window in axial (b), coronal (c), and sagittal (d) reformation. Additional details on associated hyperostosis are shown in coronal reformations (e and f). In the right nasal cavity is a smooth-bordered very low-enhancing mass centered with secondary obstruction of the osteomeatal unit on the right side and consecutive mucoid retention with complete obliteration of the maxillary cavity. The nasal septum is not destroyed. No associated tumoral calcifications are seen. The CT characteristics of the lesion are very nonspecific leading to the suggestion of an inverted papilloma. The histological preparation of the surgically resected mass revealed a hamartoma, missing the classical imaging features listed in Table 4.

cases of regrowth of residual tumor in the 14 osteoma patients who underwent resection. The 11 patients whose tumor entities were only partially resected: 1 (9.1\%) OF, 2 (18.2\%) with FD, 1 (9.1\%) with frontal osteoma, 1 (9.1\%) giant cell tumor, and the $6(54.5 \%)$ patients who were biopsied ( 3 with osteoma, 3 with FD) were stable during follow-up.

The characteristic imaging features of the BMSNs analyzed in the neuroradiological reevaluation of the CT and MRI scans of the 93 patients reviewed in this study are summarized in Table 4.

\section{Discussion}

\section{Clinical Data}

Rhinologists' experience with the rare and histologically heterogeneous group of BMSNs is sparse and limited to case reports and small case series [6]. Our retrospective analysis reaffirms the rarity of these tumors. The European position paper on endoscopic management of tumors of the nose, paranasal sinuses, and skull base provided a short but consistent overview of these pathologies [6]. 

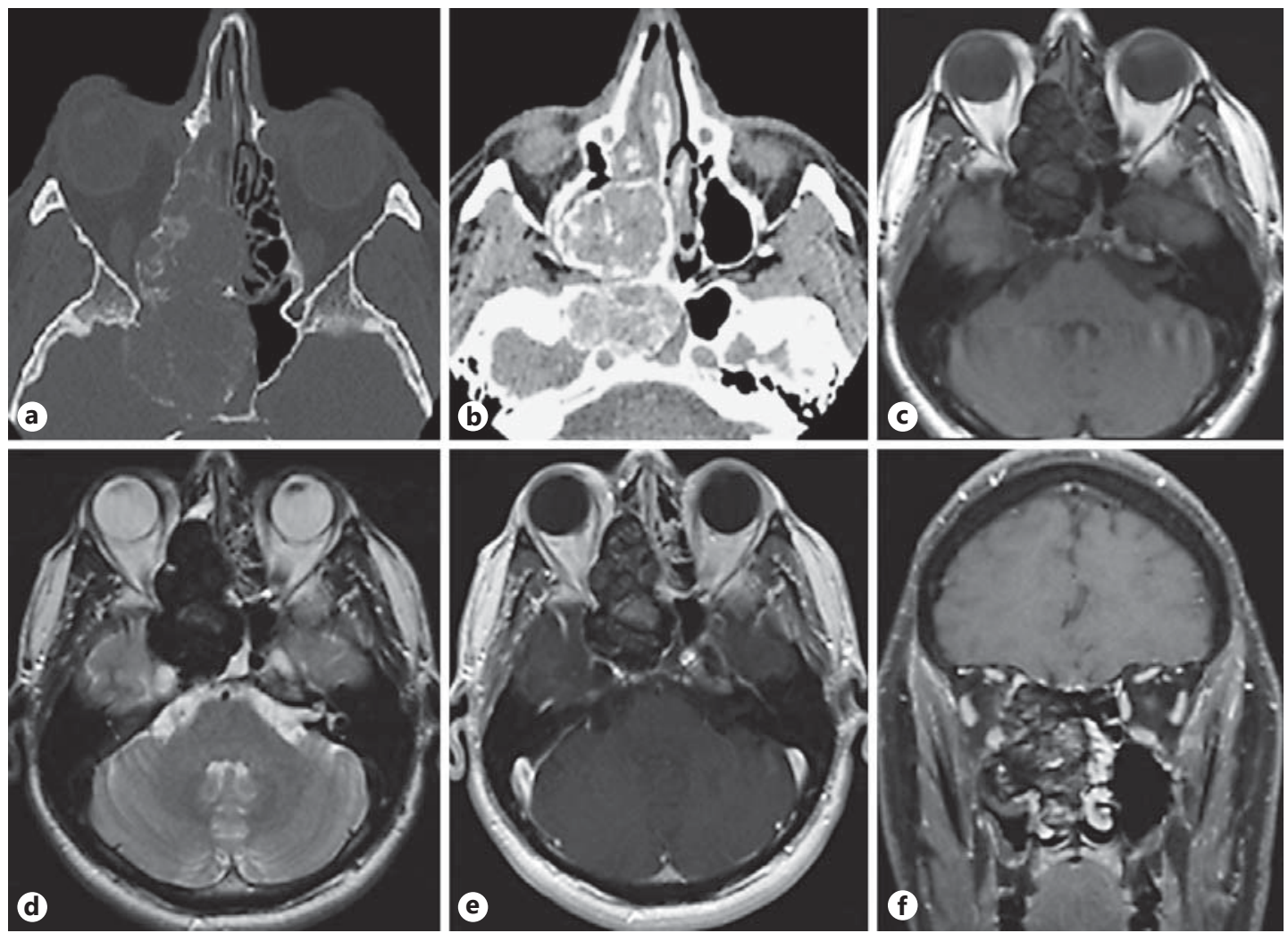

Fig. 8 The CT scan of the paranasal sinus (bone window $\mathbf{a}$ and soft tissue window $\mathbf{b}$ ) reveals a lesion appearing as an expansive homogenous mass of soft tissue density centered in the right nasal cavity with peritumoral coarse calcifications and partial calcification of the tumor mass. Note a tumor extension in the right sphenoid sinus, right ethmoidal sinus, and obstruction of the osteomeatal unit on the right. The adjacent sinus walls are extensively remodeled with scalloping of the borders. In the additional MRI, the mass was very low on the T1w (c) and T2w (d) with very low and inhomogeneous enhancement (axial, e; sagittal, f). The imaging features of this lesion show a very good match with the imaging features of a giant cell tumor, one of the rare tumor entities of the paranasal sinus.

The fibro-osseous lesions appear to be the most frequently encountered BMSN [15]. In our study, they represented $71 \%$ of the cases. Osteoma is the most frequent and best-studied benign tumor of the nose and the paranasal sinus [16] with an incidence ranging between $0.43 \%$ on plain radiographs and up to $3 \%$ on CT scans [17]. A radiological study reported in 2009 that the main area of localization may be the ethmoid sinus [18], whereas in our cohort the predominant tumor manifestation was observed in the frontal sinus (71\%) in line with Eggesbø [3]. In recent years, the endoscopic approach has taken its place in the treatment of fibro-osseous lesions of the paranasal sinuses $[19,20]$. Even in the case of a very lateral localization in the frontal sinus, the tumor may be treated endoscopically [21]. The choice of the optimal surgical strategy and an eventual decision to take an open (or endoscopic) approach largely depends on the intraoperative circumstances. Comparing the results and operative complications in our cohort between endoscopy and open resection approaches, the endoscopic approach appears to be safe and more effective. We have to take into account, however, that the endoscopically treated tumors are usually smaller and less infiltrative and adherent to the adjacent bone or soft tissue. Interestingly, we observed 2 recurrences of frontal osteomas after a combined endoscopic and open resection approach. Moreover, we noted a 

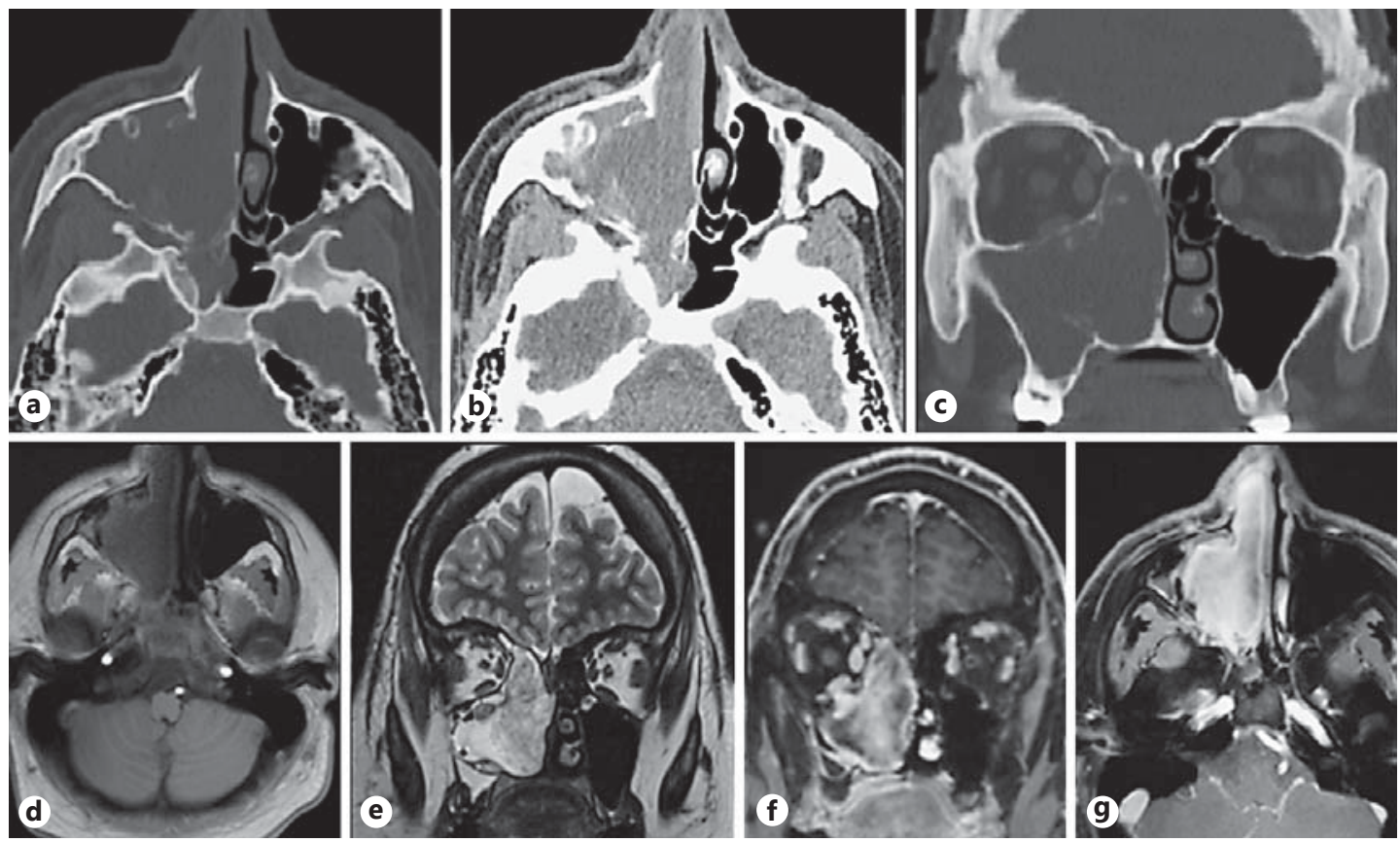

Fig. 9. The CT scan of the paranasal sinus (axial bone window, a; soft tissue window, $\mathbf{b}$; and coronal bone window, c) reveals an expansive mass of soft tissue density located in the right nasal cavity with extension into the right cavity of the maxillary sinus and consecutive expansive widening of the osteomeatal unit on the right side. No aggressive osseous destruction is seen. The MRI of the paranasal sinus shows a very low signal of the lesion on T1w (d) and T2w (e) with avid relatively homogeneous enhancement (axial, f; coronal, g). From the results of imaging, the etiology of the lesion remains unclear. A vascular tumor entity like a hemangioma or a subtype of inverted papilloma was proposed. The tumor resection and histological preparation identified a rare case of a fibromyxoma of the nasal cavity.

Fig. 10. Native CT scan of the paranasal sinus; bone (a) and soft tissue (b) window with reformation (coronal $\mathbf{c}$ and sagittal $\mathbf{d}$ ). The CT examination shows an expansive lesion located on the left side of the sphenoidal sinus with partially osteolytic and partially sclerotic tumor parts. With respect to this incidental finding, a chronic inflammatory etiology or, in the case of the known tumor history of that patient, a metastasis was proposed. The histological confirmation of the diagnosis revealed the rare case of a cholesterol granuloma. Unfortunately, no preoperative MRI scan was acquired.
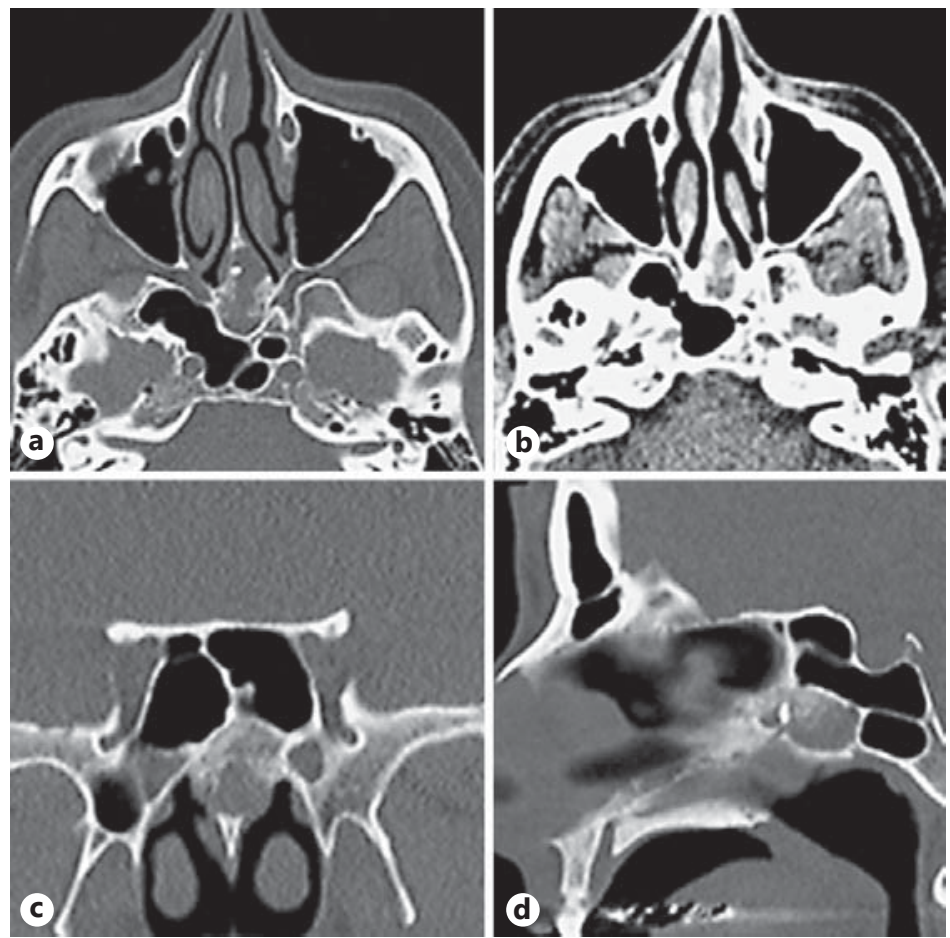
Table 3. Surgical results and follow-up findings of the study patients

\begin{tabular}{llll}
\hline & Endoscopic $(n=17)$ & Combined $(n=5)$ & Open $(n=20)$ \\
\hline $\begin{array}{l}\text { Surgical results } \\
\text { Surgical complications }\end{array}$ & 1 CRS & 1 CRS & 3 mucocele, 1 CSF leak \\
& & & $\begin{array}{l}\text { massive hemorrhage } \\
2 \text { enophthalmos }\end{array}$ \\
\hline Total tumor resection & 15 & 2 & 14 \\
\hline $\begin{array}{l}\text { Follow- } U p \\
\text { Relapse }(n=4)\end{array}$ & 1 cholesterol granuloma & 2 frontal osteomas & 1 ossifying fibroma \\
Treatment & Endoscopic debulking & 2 open resections & Open resection \\
\hline Time to relapse & 22 years & 5 and 10 months & 5 months \\
\hline Residual tumor growth $(n=6)$ & 1 angiofibroma & 1 frontal osteoma & 2 frontal osteomas \\
& & & 2 open, 1 combined $)$ \\
& & & 1 Gamma Knife, \\
Treatment & Embolisation & Open resection & 1 combined resection \\
\hline Time to revision surgery & 4 months & 7 months & 3 to 5 months for osteoma \\
& & & 1 mean 35 months for AF \\
\hline Stable residual disease $(n=5)$ & 1 ossifying fibroma & 1 frontal osteoma & 1 giant cell tumor \\
\hline Disease-free at last follow-up & 15 & 2 & 18 \\
\hline Mean follow-up time, months & 52 & 78 & 88 \\
\hline
\end{tabular}

rapid regrowth of 3 residual frontal osteomas after 1 combined procedure and 2 open tumor resections. Such cases are only rarely reported in the literature [15]. The possibility of recurrence of osteoma indicates the importance of a total tumor resection. In general, after total osteoma resection, no recurrence is documented in the reviewed literature [21]. Nevertheless, the decision to perform invasive treatment of fibro-osseous lesions should be made with caution, taking into account the possible drawbacks of radical surgery. In our retrospective study we did not observe any growth or malignant degeneration of the incidentally described osteomas; no therapy was subsequently necessary. Of the patients with FD and OF, only 2 out of $18(11.1 \%)$ underwent a gross total resection and remained stable at follow-up. Close monitoring and prompt treatment of symptomatic patients or in cases of tumor growth is prudent. Ooi et al. [22] proposed surgical treatment in cases of orbital infiltration, whereas Harvey at al. [23] recommended more aggressive management in order to preserve adjacent structures such as the dura and the orbit. No discussion arises in cases where orbital or intracranial tumor infiltration and complications have already occurred [6]. In our study, the invasion of the anterior skull base (5 patients with FD and 2 patients with OF) and the orbits (1 FD and $1 \mathrm{OF}$ ) were quite frequent, particularly in patients with FD (Table 1). The morbidity associated with radical surgical therapy has to be considered carefully. When taking into account the stabilization of FD with skeletal maturation [5], a high-risk or mutilating surgical procedure may not appear necessary until a patient shows clinical symptoms; e.g. eye movement disorders or cranial nerve dysfunction.

Soft tissue tumors in the nose and the paranasal sinus are very rare [5]. In our cohort, we observed predominantly neoplasms from fibrovascular tissues. Of that subgroup, juvenile angiofibroma (8 patients) appears to be the best-known and most studied tumor entity. In our analysis, we noted greater extension of the angiofibromas beyond the boundaries of the nasopharynx compared to the literature $[5,6]$. Prior to resection, all patients underwent a 
Anschuetz et al.: A Clinical and Radiological Approach to the Management of Benign Mesenchymal Sinonasal Tumors

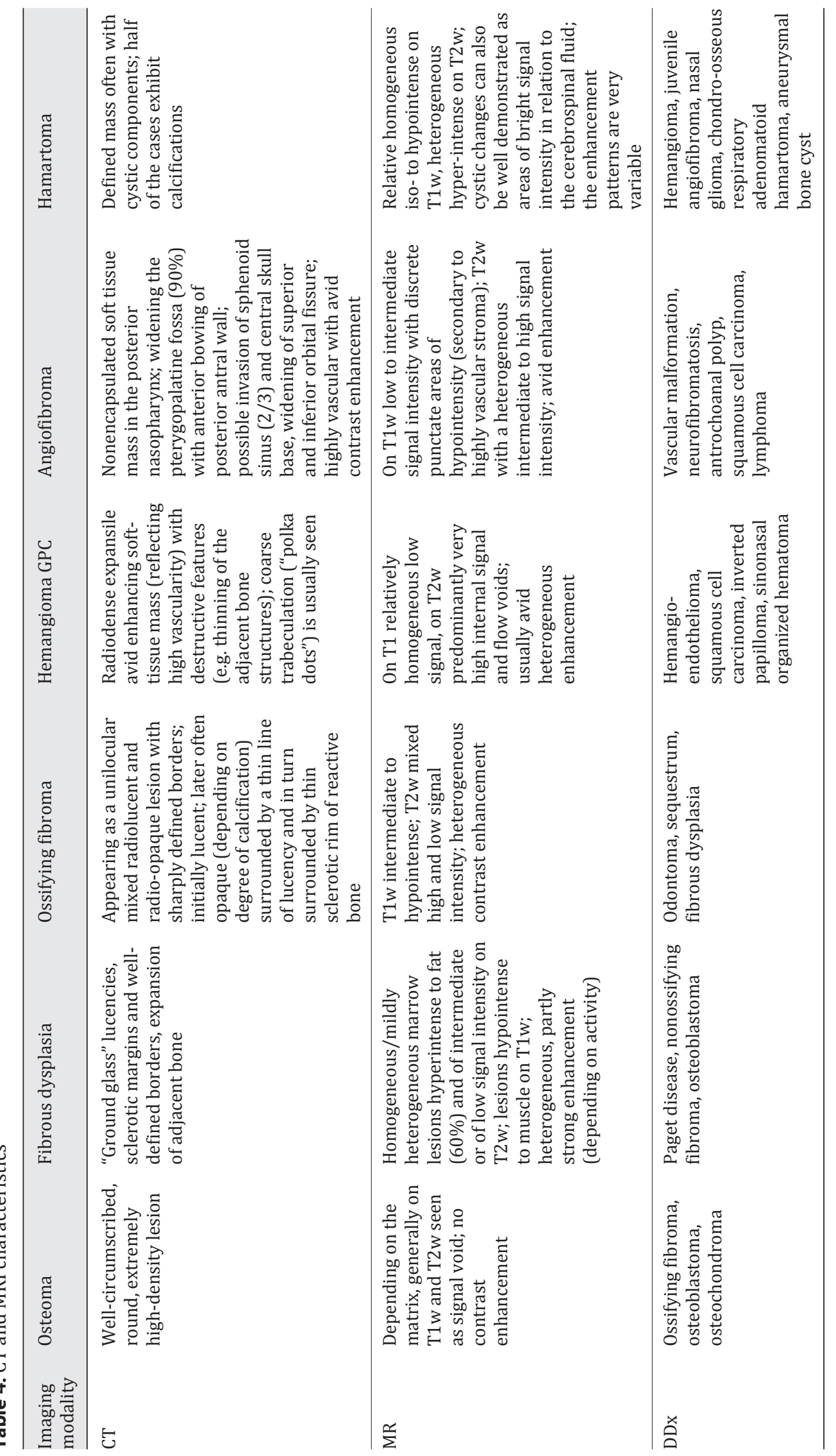


tumor embolization to reduce the intraoperative bleeding [24]. In the case of extensive or recurrent disease, the use of adjuvant-intensity modulated radiation therapy [25] or Gamma Knife radiosurgery has also been described in the literature [26, 27]. Our single case in which Gamma Knife radiosurgery was used to treat a growing residual tumor in anatomically critical areas (central skull base and pterygopalatine fossa) had good results. At follow-up, residual tumor mass was stable, confirming the findings of earlier case descriptions. The importance of long-term follow-up is illustrated in our study by 2 cases of angiofibroma with documented tumor growth 35 months after the initial operative therapy.

Hemangioma and GPCs are other rare neoplasms of the sinonasal tract [28]. For those tumors surgical and, if possible, endoscopic tumor excision is recommended [29]. In our study sample, 2 cases of hemangioma/GPC were treated with a $\mathrm{CO}_{2}$ laser and 2 patients underwent exclusive tumor embolization. Tumor embolization as treatment modality is a therapeutic option particularly in high-risk patients or for localizations which are surgically hard to reach, e.g. (para-) sagittal central midline skull base or pterygopalatine fossa.

\section{CT and MRI}

CT is the best modality for evaluating bony changes such as cortical erosion, destruction, remodeling, sclerosis, and thickening of bone. MRI is used to complement CT in order to characterize the soft tissue components of the tumor and to evaluate the extent of tumor invasion beyond the bony sinus walls. Essentially, the basic MRI protocol for imaging sinonasal tumors must include unenhanced T1- and T2-weighted imaging sequences with the aim of discriminating between different soft tissue structures: tumor or mucus or fluid-filled sinuses. The unenhanced T1-weighted series is mandatory for evaluating tumor interruption of the signal void (black) cortical bone or low tumor signal into the high-signal fatty bone marrow of the skull base. It should be noted, however, that around the sinuses, the normal bone may be too thin for proper evaluation by MRI [30]. Contrast-enhanced high-resolution, axial and coronal $\mathrm{T} 1 \mathrm{with}$ fat saturation is important for further characterization of soft tissue structures. The short tau inversion recovery or turbo inversion recovery magnitude sequence is useful for investigating bone marrow edema. MRI is also the preferred modality for detecting perivascular and perineural tumor spread as well as orbital and dural tumor invasion [31]. The characteristic CT and MRI features of BMSNs are summarized in Table 4. We are aware that a particular concern in any CT imaging of the head and neck region is the radiation dose delivered to the lens and to the thyroid gland. Depending on the imaging protocol, several studies have reported lens dose to vary widely, from 1.88 to 64 mGy [12]. These single-scan measured doses are still much lower than the 0.5-2 Gy threshold for lens damage, and very much lower than the cumulative dose of 6-14 Gy (equivalent of about 200 scans) that has been shown to increase the risk of cataract formation [12]. Thus, even patients who undergo multiple scans are at low risk for premature cataract formation. Also, with newer multidetector CT scanners, the dose has been lowered even further. This is because the newest generation of CT scanners has intrinsically better spatial and contrast resolution and more doseefficient detectors, thereby maintaining image quality at lower mAs [32-36]. In children, however, where a linear dose-response relationship is speculated, it is recommended that scans be obtained at a lower mAs setting. The dose children are exposed to may be reduced further by reconstructing coronal images from multidetector helical data, eliminating the radiation exposure involved in the direct coronal acquisition [37, 38]. Further reduction of radiation doses may also be obtained by establishing protocols in which the radiation dose delivery is tailored to the concrete clinical question [39, 40]. In our institution, we have a special age-adapted protocol for CT scans in children, according to the revised diagnostic reference values of the Bundesamt für Gesundheit (BAG) (available at: http://www.bag. admin.ch/themen/strahlung/10463/10958/). 
Although CT is ideal for assessing the osseous margins of the paranasal sinuses, the inherently superior soft-tissue resolution and multiplanar capabilities render MRI superior for assessment of soft-tissue masses and extension of infectious or neoplastic diseases beyond the paranasal sinuses [41]. MRI also better distinguishes between normal nasal mucosa or mucus retention and tumor. This is primarily because, compared with the normal sinonasal (Schneiderian) mucosa, inflamed mucosa is associated with increased submucosal edema and increased surface secretions, both of which are initially 95\% water [12]. The thin, uniformly smooth appearance of normal sinonasal mucosa can also help distinguish normal mucosa from solid tumor masses [42]. By comparison, tumor tissue is more cellular than normal or inflamed tissue and also less differentiated. This is reflected in a lower serous and mucinous content. Thus, on MRI, tumors tend to have low T1-weighted signal intensity and low to intermediate T2-weighted signal intensity. This is why T2-weighted images are the best way to distinguish inflamed mucosa from an adjacent tumor, benign or malignant [43]. If one is interested in potential intracranial extension of sinonasal disease, this will be better seen, in most cases, using contrast-enhanced MRI [44]. If the patient cannot have an MRI study (because of a pacemaker, implanted metallic fragments or because of claustrophobia), a contrast-enhanced CT scan then becomes the best available modality for assessing intracranial structures.

Although bone in the floor of the anterior cranial fossa may appear grossly intact on CT and noncontrast MRI, these modalities have limited sensitivity for early or mild intracranial spread of tumor and/or accompanying inflammation. Such conditions are best identified on contrast-enhanced, fat-suppressed, T1-weighted images. Fat suppression is needed to eliminate any high signal intensity from adjacent fat (e.g., fatty marrow) that might be confused with or obscure actual enhancement. In addition, secretions and cerebrospinal fluid, which are both hyperintense on T2-weighted imaging, could obscure adjacent pathological high signal, but are both lower in signal intensity on T1-weighted images and will not obscure enhancement [45]. As with most imaging, perfection is rarely obtained, and the distinction between tumor and some inflammatory tissues, such as granulation tissue, may still elude the clinician and neuroradiologist even with the most thoroughly performed CT and MRI studies. In such cases, a combined positron emission tomography/CT examination may provide additional useful information $[46,47]$.

\section{Conclusion}

Understanding and recognizing the spectrum of appearances of benign mesenchymal sinonasal tumors will allow improved patient assessment and clinical management at all stages including diagnosis, biopsy, staging, treatment, and prognosis. The pathognomonic neuroradiological signs of a particular tumor entity should be actively sought as the neuroradiological features may be the diagnostic clues. CT and MRI serve complementary roles in identifying the morphological details and locoregional staging of benign mesenchymal sinonasal neoplasms.

\section{Disclosure Statement}

The authors declare no conflict of interest. 
Anschuetz et al.: A Clinical and Radiological Approach to the Management of Benign Mesenchymal Sinonasal Tumors

\section{References}

1 Nicolai P, Castelnuovo P: Benign tumours of the sinonasal tract; in Flint P, Haughey BH, Lund V, Niparko J, Richardson M, Robbins KT, et al (eds): Cummings Otolaryngology-Head and Neck Surgery, ed 5. Philadelphia, Elsevier, 2010.

2 Gupta R, Moupachi SS, Poorey VK: Sinonasal masses: a retrospective analysis. Indian J Otolaryngol Head Neck Surg 2013;65:52-56.

3 Eggesbø HB: Imaging of sinonasal tumours. Cancer Imaging 2012;12:136-152.

4 Willis RA, Collins WH: Pathology of Tumours. London, Butterworth, 1948.

5 Barnes L, Eveson JW, Reichart P, Sidransky D: Tumours of the Nasal Cavity and Paranasal Sinuses. World Health Organization Classification of Tumours: Pathology and Genetics Head and Neck Tumours. Lyon, IARC Press, 2005, pp 10-80.

6 Lund VJ, Stammberger H, Nicolai P, et al: European position paper on endoscopic management of tumours of the nose, paranasal sinuses and skull base. Rhinol Suppl 2010;1-143.

7 Summers LE, Mascott CR, Tompkins JR, Richardson DE: Frontal sinus osteoma associated with cerebral abscess formation: a case report. Surg Neurol 2001;55:235-239.

8 Johnson D, Tan L: Intraparenchymal tension pneumatocele complicating frontal sinus osteoma: case report. Neurosurgery 2002; 50:878-880.

9 Mahabir RC, Szymczak A, Sutherland GR: Intracerebral pneumatocele presenting after air travel. J Neurosurg 2004;101:340-342.

10 Nabeshima K, Marutsuka K, Shimao Y, Uehara H, Kodama T: Osteoma of the frontal sinus complicated by intracranial mucocele. Pathol Int 2003;53:227-230.

11 Naraghi M, Kashfi A: Endonasal endoscopic resection of ethmoido-orbital osteoma compressing the optic nerve. Am J Otolaryngol 2003;24:408-412.

12 Fatterpekar GM, Delman BN, Som PM: Imaging the paranasal sinuses: where we are and where we are going. Anat Rec (Hoboken) 2008;291:1564-1572.

13 Das S, Kirsch CFE: Imaging of lumps and bumps in the nose: a review of sinonasal tumours. Cancer Imaging 2005;5:167-177.

14 World Medical Association Declaration of Helsinki: Ethical Principles for Medical Research Involving Human Subjects. http://jama.jamanetwork.com/article.aspx?articleid=1760318 (accessed August 12, 2015).

15 Fu YS, Perzin KH: Non-epithelial tumours of the nasal cavity, paranasal sinuses, and nasopharynx. A clinicopathologic study. II. Osseous and fibro-osseous lesions, including osteoma, fibrous dysplasia, ossifying fibroma, osteoblastoma, giant cell tumour, and osteosarcoma. Cancer 1974;33:1289-1305.

16 Eller R, Sillers M: Common fibro-osseous lesions of the paranasal sinuses. Otolaryngol Clin North Am 2006; 39:585-600.

17 Earwaker J: Paranasal sinus osteomas: a review of 46 cases. Skeletal Radiol 1993;22:417-423.

18 Erdogan N, Demir U, Songu M, Ozenler NK, Uluç E, Dirim B: A prospective study of paranasal sinus osteomas in 1,889 cases: changing patterns of localization. Laryngoscope 2009;119:2355-2359.

19 Brodish BN, Morgan CE, Sillers MJ: Endoscopic resection of fibro-osseous lesions of the paranasal sinuses. Am J Rhinol 1999;13:11-16.

20 Schick B, Steigerwald C, el Rahman el Tahan A, Draf W: The role of endonasal surgery in the management of frontoethmoidal osteomas. Rhinology 2001;39:66-70.

21 Turri-Zanoni M, Dallan I, Terranova P, Battaglia P, Karligkiotis A, Bignami M, Castelnuovo P: Frontoethmoidal and intraorbital osteomas: exploring the limits of the endoscopic approach. Arch Otolaryngol Head Neck Surg 2012;138:498-504.

22 Ooi EH, Glicksman JT, Vescan AD, Witterick IJ: An alternative management approach to paranasal sinus fibroosseous lesions. Int Forum Allergy Rhinol 2011;1:55-63.

23 Harvey RJ, Sheahan PO, Schlosser RJ: Surgical management of benign sinonasal masses. Otolaryngol Clin North Am 2009;42:353-375.

24 Glad H, Vainer B, Buchwald C, Petersen BL, Theilgaard SA, Bonvin P, Lajer C, Jakobsen J: Juvenile nasopharyngeal angiofibromas in Denmark 1981-2003: diagnosis, incidence, and treatment. Acta Otolaryngol 2007; 127:292-299.

25 Kuppersmith RB, Teh BS, Donovan DT, Mai WY, Chiu JK, Woo SY, Butler EB: The use of intensity modulated radiotherapy for the treatment of extensive and recurrent juvenile angiofibroma. Int J Pediatr Otorhinolaryngol 2000;52:261-268.

26 Álvarez FL, Suárez V, Suárez C, Llorente JL: Multimodality approach for advanced-stage juvenile nasopharyngeal angiofibromas. Head Neck 2013;35:209-213.

27 Dare AO, Gibbons KJ, Proulx GM, Fenstermaker RA: Resection followed by radiosurgery for advanced juvenile nasopharyngeal angiofibroma: report of two cases. Neurosurgery 2003;52:1207-1211.

28 Thompson LD, Fanburg-Smith JC: Update on select benign mesenchymal and meningothelial sinonasal tract lesions. Head Neck Pathol 2016;10:95-108.

29 Song CE, Cho JH, Kim SY, Kim SW, Kim BG, Kang JM: Endoscopic resection of haemangiomas in the sinonasal cavity. J Laryngol Otol 2009;123:868-872.

30 Casselman JW: The skull base: tumoral lesions. Eur Radiol 2005;15:534-542. 
Anschuetz et al.: A Clinical and Radiological Approach to the Management of Benign Mesenchymal Sinonasal Tumors

31 Maroldi R, Farina D, Borghesi A, Marconi A, Gatti E: Perineural tumor spread. Neuroimaging Clin N Am 2008; 18:413-429.

32 International Commission on Radiological Protection: Recommendations of the ICRP: publication 60; vol 21. Oxford, Pergamon Press, 1990.

33 Sillers MJ, Kuhn FA, Vickery CL: Radiation exposure in paranasal sinus imaging. Otolaryngol Head Neck Surg 1995;112:248-251.

34 Bernhardt TM, Rapp-Bernhardt U, Fessel A, Ludwig K, Reichel G, Grote R: CT scanning of the paranasal sinuses: axial helical CT with reconstruction in the coronal direction versus coronal helical CT. Br J Radiol 1998;71: 846-851.

35 Nishizawa K, Toshino-Tonari A, Matsumoto M, Aoki C, Hachiya J: Dose evaluation and effective dose estimation in radiological studies of paranasal sinuses. Radiat Prot Dosim 1998;82:271-276.

36 Bassim MK, Ebert CS, Sit RC, Senior BA: Radiation dose to the eyes and parotids during CT of the sinuses. Otolaryngol Head Neck Surg 2005;133:531-533.

37 Babbel R, Harnsberger HR, Nelson B, Sonkens J, Hunt S: Optimization of techniques in screening CT of the sinuses. AJNR Am J Neuroradiol 1991;12:849-854.

38 Mulkens TH, Broers C, Fieuws S, Termote JL, Bellnick P: Comparison of effective doses for low-dose MDCT and radiographic examination of sinuses in children. AJR Am J Roentgenol 2005;184:1611-1618.

39 Koller CJ, Eatough JP, Bettridge A: Variations in radiation dose between the same model of multislice CT scanner at different hospitals. Br J Radiol 2003;76:798-802.

40 Dubach P, Eichenberger A, Caversaccio M: Radiation dose reduction in computer assisted navigation for functional endoscopic sinus surgery - cadaver head experiments and clinical implementation. Rhinology 2010;48: 339-343.

41 Hudgins PA: Sinonasal imaging. Neuroimaging Clin N Am 1996;6:319-331.

42 Som PM: Sinonasal cavities; in Som PM, Curtin HD (eds): Head and Neck Imaging, ed 4. St. Louis, Mosby, 2003.

43 Loevner LA, Sonners AI: Imaging of neoplasms of the paranasal sinuses. Neuroimaging Clin N Am 2004;14: 625-646.

44 Som PM, Dillon W, Sze G, Biller H, Lawson W: Benign and malignant sinonasal lesions with intracranial extension: differentiation with MR imaging. Radiology 1989;172:763-766.

45 Kaufman D, Litman N, Miller M: Sinusitis induced subdural empyema. Neurology 1983;33:123-132.

46 Schoder H, Yeung HW, Gonen M, Kraus D, Larson SM: Head and neck cancer: clinical usefulness and accuracy of PET/CT image fusion. Radiology 2004;231:65-72.

47 Dubach P, et al: Image Guided Otorhinolaryngology; in Jolesz F (ed): Intraoperative Imaging and Image Guided Therapy. New York, Springer, 2014, pp 845-856. 